\title{
CREATIVIDAD Y DESARROLLO COGNOSCITIVO (UN ENFOQUE VIGOTSKYANO)
}

\author{
Beatriz Badilla Baltodano ${ }^{1}$ \\ El verdadero viaje al descubrimiento consiste \\ no es buscar nuevas tierras \\ sino en tener nuevos ojos. \\ Marcel Proust
}

\section{Resumen}

El desarrollo de la creatividad en las aulas universitarias es una necesidad en los tiempos actuales, donde los conocimientos teóricos son sólo una parte de la preparación que el futuro profesional debe tener. Los y las docentes deben considerar que la creatividad es algo que se puede fomentar y estimular en el acto educativo, por lo que tiene un importante papel que cumplir en este campo. En el presente trabajo, se hace el planteamiento del desarrollo de la creatividad basado en los esquemas establecidos por Liev Vigotsky, quien con su aporte ha sentado las bases para desarrollar estas habilidades.
Palabras claves: Creatividad, Vigotsky, desarrollo cognoscitivo.
Abstract
Development of creativity in university classrooms is of great need in present ti- mes, when theoretical knowledge is only part of the formation that a future pro- fessional must have. University teachers must be aware that creativity can be fo- mented and stimulated during college education, therefore, it is an important part of his/her role as an educator. In the present work, development of creativity is explained, based on the schemes es- tablished by Liev Vigotsky who has es- tablished the foundations for developing these abilities.

1 Docente e investigadora. Labora en la Facultad de Farmacia de la Universidad de Costa Rica. Máster en Farmacología del Sistema de Estudios de Postgrado de la Universidad de Costa Rica. Actualmente es la coordinadora de la Coordinación de la Comisión de Currículum de la Facultad de Farmacia y es estudiante de la Maestria en Currículum y Docencia Universitaria. Su experiencia docente e investigativa se inscribe en el campo de la farmacología y recientemente se introduce en el campo de la didáctica y pedagogía universitaria. E-mail: bbadilla@cariari.ucr.ac.cr 
Keywords: Creativity. Vigosky, cognitive development.

T a creatividad, como acto humano es un proceso cognitivo y en su prístino Lestado, una muestra del procesamiento que el ser humano hace de la información. En el siglo XXI, cuando el conocimiento es uno de los principales valores de la sociedad, la creatividad debe promoverse y desarrollarse en las instituciones educativas usando las herramientas pedagógicas adecuadas y centrándose en la producción de la cultura, la ciencia y la tecnología, que eleve el nivel de los habitantes de nuestro país, en la capacidad para resolver problemas.

Educar en la creatividad, es educar para el cambio y formar personas ricas en originalidad, flexibilidad, visión futura, iniciativa, confianza, amantes de los riesgos y listas para afrontar los obstáculos y problemas que se les van presentado en su vida académica y cotidiana; además de ofrecerles herramientas para la innovación (De Bono, 1994). Tal como lo manifiesta De Bono (1996), una de las tareas para formar profesionales en condiciones suficientes, como para que puedan desarrollarse en forma integral, es fundamentalmente hacer que nuestros egresados de las universidades abandonen su clásica actividad de buscadores de trabajo y asuman la de creadores de trabajo.

De las teorías acerca de la naturaleza de la creatividad, la humanista parece estar más cercana a la aplicación en la educación. El gran aporte de esta teoría constituye el estudio de la creatividad a partir de la personalidad del sujeto creador. El acto creativo se produce por la motivación, la asimilación consciente y el esfuerzo dirigido hacia un determinado problema, de forma, que luego se interiorice al subconsciente para producir una lucha interna en este nivel, del cual surge el "insight" o intuición.

Este proceso comienza con el contacto del individuo con la realidad, a través de la implicación y el compromiso, manifestándose en la producción de lo nuevo, para la satisfacción de las necesidades individuales, realizándose de forma estimulante y gratificante. Maslow (1982) define el término de autorrealización como tendencia a llegar a ser todo lo que se es capaz de ser. Sin embargo, Carl Rogers (1983) considera que aparte de los rasgos personológicos propicios para el desarrollo de la creatividad, como apertura a la experiencia, capacidad de autoevaluación y regulación interna, capacidad de jugar con elementos y conceptos, debe existir algo observable, los productos deben ser construcciones novedosas, considerando además que el efecto de 
las relaciones sociales, según sea el caso, propiciarán o bloquearán el desarrollo creativo (Goñi, 2000).

La creatividad puede ser desarrollada a través del proceso educativo, que favorecen las potencialidades y consiguen una mejor utilización de los recursos individuales y grupales dentro del proceso de enseñanza-aprendizaje. La concepción acerca de una educación creativa parte del planteamiento de que la creatividad está ligada a todos los ámbitos de la actividad humana y es el producto de un devenir histórico social determinado.

La creatividad es algo que se puede construir, fomentar o moldear y los y las docentes son quienes deben poner los elementos necesarios para que el y la estudiante asciendan y llegue al acto creativo.

Para el abordaje de la gestión de la creatividad, podemos servirnos de los conceptos teóricos en torno a los que se ha organizado la propuesta de Vigotsky, en especial en la influencia de la actividad instrumental y la interacción social en el estudio de los procesos psicológicos superiores, en las relaciones entre pensamiento y lenguaje, en la influencia de las variables sociohistóricas y en la Zona de Desarrollo Próximo (Carretero, 1998).

Usualmente se entiende por andamiaje una situación de interacción entre un sujeto experto o más experimentado en un dominio y otro novato o menos experto, en la que el formato de la interacción tiene por objeto que el sujeto menos experto se apropie de forma gradual del saber experto. El formato debería contemplar que el novato participe desde el comienzo en una tarea reconocida como compleja, aunque su participación inicial sea sobre aspectos parciales o locales de la actividad global y aún cuando se requiere del apoyo del sujeto más experto (Barquero, 2004).

El andamiaje, por lo tanto, requiere de una actividad colaborativa y esta estructura alude a un tipo de ayuda que debe tener como estructura su propio desmontaje progresivo. Griffin y Cole (1984) han criticado el andamiaje al indicar que deja abierto el problema de la creatividad en el estudiante y un sesgo fuertemente teleológico en el modelo general de desarrollo, toda vez que los logros del más experto regulan las adquisiciones de los estudiantes.

Según el autor ruso, todos los procesos superiores o complejos de la conducta, ya estén relacionados con el pensamiento, el lenguaje o la actividad motora, tienen un carácter mediacional. Consisten en la utilización no sólo de estímulos del medio, sino de los estímulos o recursos internos que el sujeto va construyendo a lo largo de su desarrollo, es decir, todas las funciones superiores se originan como relaciones entre los seres humanos. 
La adquisición de los instrumentos cognitivos depende, en gran medida, del medio social en el que se desarrolla o educa el sujeto. El ser humano, en el momento de su nacimiento, es heredero de toda su evolución filogenética, pero el producto final de su desarrollo estará en función de las características del medio social que lo rodea. La creatividad del Homo sapiens estuvo determinada por sus necesidades y por su entorno y no podría compararse con la del homo industrializado en donde sus necesidades y de estímulos son distintos.

Los trabajos de Vigotsky incluían el estudio de procesos perceptivos, de clasificación, deducciones e inferencias, razonamiento y solución de problemas, elementos necesarios para la creatividad; sus conclusiones muestran de forma clara que todos estos aspectos se ven influidos por el grado de experiencia educativa y social que tenga el sujeto.

La enorme influencia de la interacción social se ve complementada con los aspectos microsociales con el concepto vigotskyano de la Zona de Desarrollo Próximo. Este concepto se ha orientado al análisis de los sistemas de interacción en donde se producen los progresos del desarrollo subjetivo $\mathrm{y}$, en términos generales, puede entenderse como la estructura de actividad conjunta en cualquier contexto donde hay participantes que ejercen responsabilidades diferenciales en virtud de su distinta pericia (Cole, 1989).

Este agente promotor o participante con más pericia no siempre tiene que ser otra persona; este papel lo pueden tomar las herramientas culturales. Cuando se habla de creatividad, se hace énfasis en el ambiente estimulante, lleno de herramientas para la creación, es decir, una zona en donde se potencien las capacidades de las y los estudiantes hacia la meta creadora que coincide con la Zona de Desarrollo Próximo al mejor estilo de Vigotsky. El aprendizaje, en el ambiente creativo, despertará una serie de procesos evolutivos intemos capaces de operar sólo cuande el alumno o la alumna esté en el entorno social adecuado.

Wetheimer, Mednick, Khler y Koesdtler, principales representantes de la teoría perceptual de la creatividad, indican que el pensamiento productivo se manifiesta ante la necesidad producida por un estímulo. Los ambientes creativos deberán presentar esos estímulos desarrolladores de la creatividad, que planteen los elementos de rompimiento de los esquemas que produzcan transformaciones radicales a la realidad, se procuren la proyección de enfoque y procesos, la imagen nueva y la certeza de éxito respecto a la creación.

Hablar de creatividad en los albores del presente siglo, es tocar un tema polémico debido, fundamentalmente, a las diversas tendencias y posiciones desde las que se enfoca, pudiendo también considerarse como esencia misma 
y característica de la humanidad. La invención de las primeras herramientas, armas y utensilios, así como la creación del idioma y del lenguaje, marcan un hito fundamental y decisivo del hombre en su dimensión creadora; dimensión que siempre ha ido de la mano con su desarrollo aun en las épocas del oscurantismo, pese a las restricciones de carácter místico y religioso y al excesivo dogmatismo como una necesidad humana de resolver sus problemas. La creatividad es un proceso influenciado por múltiples elementos. Siendo reduccionistas, hemos esbozado sólo algunos de ellos que consideramos esenciales.

Quizás el motivo del asunto creativo, desde nuestra perspectiva, está en preguntarnos, ¿qué influjo puede tener el educador sobre la creatividad de sus estudiantes?, ¿se puede afirmar que donde haya un educador creativo habrá creatividad? Carl Rogers (1983) ha establecido que el propósito del educador es la facilitación del aprendizaje y, en buena teoría, conocemos que en la relación educador-educando, se produce la transferencia, a partir de la cual se elaboran las hipótesis sobre la inducción de actitudes y modos de enfocar la realidad que tiene lugar como flujo hacia los y las estudiantes. El mediador estereotipado, repetidor de fórmulas y esquemas, es evidente que obtendrá por respuesta este tipo de pensamientos, mientras el poseedor de una mente capaz de disociar o de reestructurar los contenidos y la realidad, cuenta con un pensamiento crítico; el y la docente que eduquen en libertad y con motivación podrán esperar esta misma actitud en sus estudiantes. Gran responsabilidad recae, entonces, sobre los y las docentes.

Debemos concluir indicando que una de las tareas obligadas de los educadores y las educadoras es construir una condición necesaria para el desarrollo de modelos pedagógicos diseñados para educar y desarrollar la capacidad creativa de los individuos dentro, pero especialmente fuera de los espacios pedagógicos. Empezar acciones que contribuyan al desarrollo de la creatividad en las aulas universitarias, es urgente e indispensable. La creatividad es una condición necesaria para el desarrollo de la humanidad, para la calidad de lo humano. Seremos más humanos cuanto más creativos seamos (Csikszentmijaly, 1998).

La mayor riqueza de las naciones, como también de los individuos, es su capacidad mental y, en especial, la intelectual (inteligencia y creatividad). La anterior afirmación se fundamenta, entre otras razones, en el hecho de que nuestra civilización, en los últimos tiempos, ha invertido grandes cantidades de capital y esfuerzo en promover las potencialidades de los individuos. En nuestros días, ninguna cosa tiene tanto valor como la innovación en todos los campos del saber. La importancia de la creatividad para el porvenir y la 
competitividad económica de las naciones es tanta, que los nuevos índices de desarrollo no son exclusivamente econométricos, sino inteligentes (Proyecto Estado de la Nación, 2003).

Por ello, en la actualidad, quienes miden el desarrollo hacen uso de indicadores tales como el número de científicos, de tecnólogos, de innovaciones y de publicaciones de un país. Cobra una nueva dimensión la producción intelectual; los indicadores humanos valen y dicen muchísimo más acerca de un país, que la extensión de sus vías o el número de aviones que posea (De Zubiria y De Zubiria, 1994). Por todo lo anterior, las naciones, sobre todo las del tercer mundo, deben dar pasos veloces y seguros para formar individuos inteligentes y creativos, capaces de plantear soluciones creativas a la problemática particular de América Latina.

Las instituciones educativas públicas y privadas son los instrumentos con que cuentan los gobiernos para abordar esta situación y es en su interior, donde debemos cultivar y proyectar las potencialidades intelectuales de los estudiantes.

Jerone Bruner, quien propuso la teoría del aprendizaje por descubrimiento, establece que el individuo creativo es aquel capaz de revolucionar los sistemas de ideas tradicionales a partir de los sistemas culturalmente establecidos (1966). Se han hecho diferentes aproximaciones a los principios ideológicos que sustentan el concepto de creatividad. En 1852, el Marqués de Casajara, Juan Manuel de Berriozabal se refiera a la creatividad definiéndola como "la capacidad superior del ser humano que puede ser comprendida como entendimiento aventajado" (Berriozabal, 1852).

La verdadera dimensión de la relevancia de la creatividad, desde un punto de vista científico, se dio a partir de 1950, cuando J. Guilford destacó la importancia de considerar la creatividad como una categoría dentro de los estudios e investigaciones de la psicología (Martínez, 1997). Este mismo autor indicó que la creatividad en su estado más puro es conciencia pura que transciende a la persona, transforma la realidad o la crea (Guilford, 1950).

Diversas teorías psicológicas, a partir de sus respectivos principios filosóficos, han generado distintas concepciones acerca de la naturaleza de la creatividad, entre las que se encuentran la concepción de creatividad como proceso, como producto, a partir de rasgos personales, de las condiciones del medio y la concepción personológica integral. Si bien hasta hace poco tiempo se le consideraba a cada una de estas teorías en forma aislada y algunas veces antagónica, la visión actual, desde un enfoque sistémico, es que han 
llegado a complementarse, pese a las diferencias de carácter filosófico. Esto ha significado una explicación mucho más completa del fenómeno y la aparición de indicadores descriptivos múltiples para su consideración en diversas investigaciones acerca de la creatividad, especialmente, en la educación.

La creatividad, como acto humano, es un proceso cognitivo y en su prístino estado, una muestra del procesamiento que el ser humano hace de la información. En el siglo XXI, cuando el conocimiento es uno de los principales valores de la sociedad, la creatividad debe promoverse y desarrollarse en las instituciones educativas usando las herramientas pedagógicas adecuadas y centrándose en la producción de la cultura, la ciencia y la tecnología, que eleve el nivel de los habitantes de nuestro país, en la capacidad para resolver problemas. 


\section{Referencias}

Barquero, R. (2004) Vigotsky y el aprendizaje escolar. (4ta. ed.). Buenos Aires: AIQUE.

Berriozabal, J. M. (1852). El talento bajo todos sus aspectos y relaciones. Madrid: NICOLÁS DE CASTRO Y PALOMINO.

Bono, E. (1994). El pensamiento creativo. Barcelona: PIADÓS IBÉRICA S.A.

Bono, E. (1996). Cultura, educación y desarrollo. Conferencia sobre políticas y educación para la transformación de la educación superior en América Latina y el Caribe. La Habana, Cuba.

Bruner, J. (1966). Toward a theory of instruction. Cambrige, MA: BELKAPP PRESS.

Carretero, M. (1998). Introducción a la psicología cognitiva. Buenos Aires: AIQUE.

Cole, M. (1989). "The Zone of Proximal Development: where culture and cognition create each odder". En Wertsch, J. Voices on the mind. The sociocultural approach to mediated action. Cambridge. Harvard University.

Csikszentmijaly, M. (1998). Creatividad. México, D.F.: PAIDÓS.

Goñi, A. (2000). Desarrollo de la creatividad. San José, Costa Rica: UNED.

Griffin, P. y Cole, M. (1984). "Current activity for the future: the zo-ped". En Rogoff, B. y J. V. Wertsch (eds.). Children's Learning in the "Zone of Proximal Development". New Directions for Child Development, núm. 23, JosseyBass, San Francisco.

Guilford, J. P. (1950). "Creativity”. American Psychologist, 5, pp. 444-454.

Martínez, L. (1997). Educación y creatividad: algunas tendencias. Conferencia pronunciada en la sesión inaugural del VI Taller Internacional "La educación hacia el siglo XXI”, Instituto Pedagógico Latinoamericano y Caribeño (IPLAC).

Maslow, A. (1982). La personalidad creadora. Barcelona: CAÍDOS.

Proyecto Estado de la Nación en Desarrollo Humano Sostenible. (2003). Noveno Informe. San José, Costa Rica.

Rogers, C. (1983). Libertad y creatividad en la educación. Barcelona: PIADÓS.

Zubiria, M. y Zubiria, A. (1994). Operaciones intelectuales y creatividad. Colombia: FONDO DE PUBLICACIONES BERNARDO HERRERA MERINO. 